\title{
Strings in extremal BTZ black holes
}

\author{
Jamie Parsons* and Simon F. Ross ${ }^{\dagger}$ \\ Centre for Particle Theory \& Department of Mathematical Sciences, \\ Durham Univerity, South Road, Durham DH1 3LE, United Kingdom.
}

October 30, 2018

\begin{abstract}
We study the spectrum of the worldsheet theory of the bosonic closed string in the massless and extremal rotating BTZ black holes. We use a hyperbolic Wakimoto representation of the $S L(2, \mathbb{R})$ currents to construct vertex operators for the string modes on these backgrounds. We argue that there are tachyons in the twisted sector, but these are not localised near the horizon. We study the relation to the null orbifold in the limit of vanishing cosmological constant. We also discuss the problem of extending this analysis to the supersymmetric case.
\end{abstract}

*J.D.Parsons@durham.ac.uk

†S.F.Ross@durham.ac.uk 


\section{Introduction}

The Banados-Teitelboim-Zanelli (BTZ) black hole [1, 2] is a very useful laboratory for exploring aspects of black holes and geometry in a simplified setting, as the geometry is simply an orbifold of $\mathrm{AdS}_{3}$. In particular, if we consider an $\mathrm{AdS}_{3} \times S^{3}$ solution in string theory supported by NS-NS fluxes, the spectrum of perturbative string theory on the BTZ black hole can easily be determined using standard orbifold techniques. This problem has been extensively investigated for non-extremal BTZ black holes, which are orbifolds of $\mathrm{AdS}_{3}$ along a hyperbolic generator [3, 4, 5, 6, 17, 8]. The twisted sector states for the orbifold are obtained using a parafermionic representation of the current algebra and a twist operator construction which are based on earlier work on the long string sectors of global $\mathrm{AdS}_{3}$ [9] and closely related to the work on the elliptic orbifold in [10]. The extremal black holes, which are orbifolds along a parabolic generator, are less studied (although the winding sectors were considered in [11], where the relevance of the hyperbolic Wakimoto representation of the currents used in [4] was recognized). Since the extremal cases are a different class of orbifolds from the non-extremal cases, they will require separate consideration. From the worldsheet point of view, the parafermionic representation used in the non-extremal case are no longer appropriate, and we need to find a new representation of the vertex operators which diagonalises the angular momentum in the extremal BTZ black hole.

This paper addresses this problem, finding an explicit set of vertex operators for the untwisted and twisted sectors for the bosonic string on the zero-mass and extremal rotating BTZ black holes. We consider an $\mathrm{AdS}_{3} \times S^{3}$ geometry supported by NS-NS flux, corresponding to an F1-NS5 system compactified on a Ricci-flat internal manifold. The world-sheet theory is a CFT with an $\widehat{S L(2, \mathbb{R})_{k}} \times \widehat{S U(2)}{ }_{k}$ current algebra, with the level $k$ being set by the NS-NS flux. We will discuss the bosonic string in detail; the problem of extending our analysis to the superstring will be discussed at the end. We want to work in a parabolic basis for $S L(2, \mathbb{R})$, which diagonalises the combination of generators corresponding to the momentum along the compact circle in zero-mass BTZ. Here we show that the hyperbolic Wakimoto representation introduced in [4, provides an appropriate representation of the $S \widehat{L(2, \mathbb{R})_{k}}$ current algebra. This representation has the advantage that the expression for the vertex operators is more explicit than in the parafermionic representation used in the non-extremal case.

We apply this calculation of the spectrum to study the tachyons in this background. Tachyons in the non-extremal non-rotating BTZ black hole were studied in [8], as an explicit example of the kind of quasi-localised closed string tachyons discussed in [12] The idea is that if we consider string theory compactified on a circle, when the size of the circle is smaller than the string length $\ell_{s}$, there are tachyonic winding modes. If the size of this circle varies over some base space, one heuristically expects a tachyon which is confined to the region where the size of the circle $\leq \ell_{s}$. It was found in [8] that there is a tachyon in the twisted sector NS-NS ground state if the size of the circle at the black hole horizon is smaller than the string scale, $\sqrt{k} r_{+} \leq \ell_{s}$. However, this tachyon is found not to be localised in the near-horizon region, due to the coupling to the NS-NS field. As the zero-mass BTZ

\footnotetext{
${ }^{1}$ As BTZ arises as the near-horizon limit of a charged black string, it is directly related to the examples discussed in [13, 14]. Other examples with a quasi-localised tachyon include [15, 16].
} 
black hole is the limit as $r_{+} \rightarrow 0$, we would expect that in this case, the NS-NS ground state in twisted sectors should always be tachyonic, and using the explicit representation of the spectrum we obtain, that is indeed what we find. We also extend the analysis to the rotating case, showing that tachyons arise in the twisted sectors if $\sqrt{k} r_{+} \leq 2 \ell_{s}$, as in the previous discussion of the non-rotating non-extremal case. The study of tachyons in the $M=0$ BTZ black hole has a couple of advantages over the previous non-extremal case. Firstly, the expressions for the vertex operators in the Wakimoto representation are more explicit than the parafermionic representation used in the previous case. Secondly, the geometry has a causal Killing vector everywhere, so issues of tachyon condensation could be addressed in the $M=0 \mathrm{BTZ}$ black hole without having to deal with the complications of studying the behaviour on a time-dependent background geometry in the region behind the horizon. We will not address the question of the condensation of the tachyon, which remains a challenging direction for future work.

In section 2, we review aspects of the $M=0$ BTZ black hole. In section 3, we introduce the hyperbolic Wakimoto representation of the current algebra, and use it to construct vertex operators for the untwisted sector states. We then introduce a twist operator enforcing the orbifold condition, and use it to obtain the twisted sector vertex operators. We discuss the condition for a tachyon to exist in the spectrum, and argue that the NS-NS ground states in the twisted sector are tachyonic, as expected. In section 4, we discuss the flat space limit, taking $k \rightarrow \infty$ while focusing on the neighbourhood of the singularity. In this limit, the zero mass BTZ black hole reduces to the null orbifold of flat space [17, 18].

In section 5, we extend the analysis to the extremal rotating BTZ black hole. We show that the relevant orbifold action is chiral, with the action on left-movers the same as for the zero-mass black hole while the action on right-movers is the same as for the non-zero mass black hole. We can thus construct appropriate vertex operators by combining the previous results for these two cases. We show that the resulting set of vertex operators for twisted sectors is mutually local, and argue that a tachyon appears when $\sqrt{k} r_{+} \leq 2 \ell_{s}$, as expected.

In section 6 , we discuss the extension of our results to the superstring. The main open problem is to find a representation of the spin fields which diagonalises the action of the spacetime angular momentum. Without such a representation, we cannot explicitly construct vertex operators corresponding to the modes which survive the orbifold projection in the NS$\mathrm{R}$ and $\mathrm{R}-\mathrm{R}$ sectors. The final section summarises the paper and considers possible routes for further investigation.

\section{$2 \quad \mathrm{AdS}_{3}$ worldsheet theory}

Bosonic string theory on $A d S_{3}$ is described by the $S L(2, \mathbb{R})$ WZW model with action

$$
S_{W Z W}=\frac{k}{8 \pi \alpha^{\prime}} \int d^{2} \sigma \operatorname{Tr}\left(g^{-1} \partial_{a} g g^{-1} \partial^{a} g\right)+\frac{i k}{12 \pi} \int \operatorname{Tr}\left(g^{-1} d g \wedge g^{-1} d g \wedge g^{-1} d g\right)
$$

where $g$ is the $S L(2, \mathbb{R})$ group element, $k$ is the level, and the second integral runs over a three-dimensional manifold whose boundary is the two-dimensional worldsheet (see e.g. [19] for a nice discussion of strings on $\mathrm{AdS}_{3}$ ). Henceforth we will set $\alpha^{\prime}=1$, so we work in units of the string length. The AdS length scale is then $\ell=\sqrt{k}$. The WZW model is invariant 
under the action

$$
g(z, \bar{z}) \rightarrow \omega(z) g(z, \bar{z}) \bar{\omega}(\bar{z})^{-1}
$$

which leads to a set of conserved world-sheet currents,

$$
\mathcal{J}(z)=\mathcal{J}_{a} \tau^{a}=-\frac{k}{2} \partial g g^{-1}, \quad \overline{\mathcal{J}}(\bar{z})=\overline{\mathcal{J}}_{a} \tau^{a}=-\frac{k}{2} g^{-1} \bar{\partial} g
$$

where the generators of $S L(2, \mathbb{R})$ are given by

$$
\begin{aligned}
\tau^{1} & =\frac{i}{2} \sigma^{3}=\frac{1}{2}\left(\begin{array}{cc}
i & 0 \\
0 & -i
\end{array}\right), \\
\tau^{2} & =\frac{i}{2} \sigma^{1}=\frac{1}{2}\left(\begin{array}{cc}
0 & i \\
i & 0
\end{array}\right), \\
\tau^{3} & =\frac{1}{2} \sigma^{2}=\frac{1}{2}\left(\begin{array}{cc}
0 & -i \\
i & 0
\end{array}\right) .
\end{aligned}
$$

These satisfy the algebra

$$
\left[\tau^{a}, \tau^{b}\right]=i \epsilon^{a b}{ }_{c}^{c}
$$

where $\epsilon^{123}=1$, and the index is lowered with the metric $\eta_{a b}=\operatorname{diag}(1,1,-1)$. It will later be convenient to introduce the combinations $\tau^{ \pm}=\tau^{3} \pm \tau^{2}$. Note that this choice of notation doesn't correspond to the usual conventions for raising and lowering operators: we have the commutation relations $\left[\tau^{1}, \tau^{+}\right]=-i \tau^{+},\left[\tau^{1}, \tau^{-}\right]=i \tau^{-},\left[\tau^{+}, \tau^{-}\right]=2 i \tau^{1}$.

Using the Ward identity

$$
i \delta A(w, \bar{w})=\oint_{w} \frac{d z}{2 \pi i} \epsilon_{a} \mathcal{J}^{a} A(w, \bar{w})+\oint_{w} \frac{d \bar{z}}{2 \pi i} \bar{\epsilon}_{a} \overline{\mathcal{J}}^{a} A(w, \bar{w})
$$

we can find the OPEs for the currents. They are

$$
\mathcal{J}^{b} \mathcal{J}^{c}=\frac{i \epsilon_{a}^{b c} \mathcal{J}^{a}}{z-w}+\frac{\frac{k}{2} \eta^{b c}}{(z-w)^{2}}, \quad \overline{\mathcal{J}}^{b} \overline{\mathcal{J}}^{c}=-\frac{i \epsilon_{a}^{b c} \overline{\mathcal{J}}^{a}}{\bar{z}-\bar{w}}+\frac{\frac{k}{2} \eta^{b c}}{(\bar{z}-\bar{w})^{2}}
$$

where $\epsilon^{a b c}$ is the totally antisymmetric tensor, with $\epsilon^{123}=1$, and $\eta^{a b}$ is the metric defined by $\eta^{a b}=\operatorname{diag}(1,1,-1)$. There is a relative minus sign between the OPEs for the left and the right moving currents, as noted in [10]. This minus sign can be fixed using a relabelling process, setting $J^{a}=\mathcal{J}^{a}, \bar{J}^{1}=\overline{\mathcal{J}}^{1}, \bar{J}^{3}=\overline{\mathcal{J}}^{3}, \bar{J}^{2}=-\overline{\mathcal{J}}^{2}$. The OPEs for both the left and right moving sectors are then identical for the new currents,

$$
J^{b} J^{c}=\frac{i \epsilon_{a}^{b c} J^{a}}{z-w}+\frac{\frac{k}{2} \eta^{b c}}{(z-w)^{2}}, \quad \bar{J}^{b} \bar{J}^{c}=\frac{i \epsilon_{a}^{b c} \bar{J}^{a}}{\bar{z}-\bar{w}}+\frac{\frac{k}{2} \eta^{b c}}{(\bar{z}-\bar{w})^{2}} .
$$

Assuming the currents have trivial monodromies, they will have a mode expansion

$$
J^{a}=\sum_{n \in \mathbb{Z}} z^{-n-1} J_{n}^{a} \quad \bar{J}^{a}=\sum_{n \in \mathbb{Z}} \bar{z}^{-n-1} \bar{J}_{n}^{a} .
$$

The commutation relations for these modes are then

$$
\left[J_{n}^{a}, J_{m}^{b}\right]=i \epsilon_{c}^{a b} J_{m+n}^{c}+\frac{k}{2} n \eta^{a b} \delta_{m+n, 0},
$$

and similarly for the $\bar{J}^{a}$. In particular the zero modes form an $S L(2, \mathbb{R}) \times S L(2, \mathbb{R})$ subalgebra, corresponding to the spacetime isometries. 


\subsection{Zero mass black hole}

The $M=0$ BTZ black hole corresponds to writing the space in Poincaré coordinates and making an identification. In these coordinates the $S L(2, \mathbb{R})$ group element is

$$
g=\left(\begin{array}{cc}
\frac{1}{z} & \frac{(t+x)}{z} \\
\frac{(x-t)}{z} & \frac{\left(x^{2}+z^{2}-t^{2}\right)}{z}
\end{array}\right),
$$

so the metric is

$$
d s^{2}=\frac{k}{z^{2}}\left(-d t^{2}+d z^{2}+d x^{2}\right)
$$

and the NSNS 2-form field is

$$
B=\frac{k}{z^{2}} d t \wedge d x
$$

In Poincaré coordinates, the currents are

$$
\begin{aligned}
J^{1} & =-i k\left[(\partial x+\partial t) \frac{(x-t)}{z^{2}}+\frac{\partial z}{z}\right], \\
J^{2} & =i k\left[-\frac{(x-t)}{z} \partial z+\frac{(\partial x-\partial t)}{2}+\frac{(\partial x+\partial t)}{2 z^{2}}\left(2 t x+1-x^{2}-t^{2}\right)\right], \\
J^{3} & =-i k\left[-\frac{(x-t)}{z} \partial z+\frac{(\partial x-\partial t)}{2}+\frac{(\partial x+\partial t)}{2 z^{2}}\left(2 t x-1-x^{2}-t^{2}\right)\right],
\end{aligned}
$$

and

$$
\begin{aligned}
\bar{J}^{1} & =-i k\left[(\bar{\partial} x-\bar{\partial} t) \frac{(x+t)}{z^{2}}+\frac{\bar{\partial} z}{z}\right], \\
\bar{J}^{2} & =-i k\left[-\frac{(x+t)}{z} \bar{\partial} z+\frac{(\bar{\partial} x+\bar{\partial} t)}{2}+\frac{(\bar{\partial} x-\bar{\partial} t)}{2 z^{2}}\left(-2 t x+1-x^{2}-t^{2}\right)\right], \\
\bar{J}^{3} & =i k\left[-\frac{(x+t)}{z} \bar{\partial} z+\frac{(\bar{\partial} x+\bar{\partial} t)}{2}+\frac{(\bar{\partial} x-\bar{\partial} t)}{2 z^{2}}\left(-2 t x-1-x^{2}-t^{2}\right)\right] .
\end{aligned}
$$

To relate the spacetime energy and momentum to these currents, we consider infinitesimal time and space translations. For the time-translation, the infinitesimal transformation is

$$
\delta_{(t)} g \equiv i \epsilon_{(t)}(z) g-i g \bar{\epsilon}_{(t)}(\bar{z})=\frac{\partial g}{\partial t} \delta t
$$

where

$$
i \epsilon_{(t)}(z)=\left(\begin{array}{cc}
0 & 0 \\
-1 & 0
\end{array}\right) \delta t, \quad i \bar{\epsilon}_{(t)}(\bar{z})=\left(\begin{array}{cc}
0 & -1 \\
0 & 0
\end{array}\right) \delta t .
$$

Thus, in terms of the $S L(2, \mathbb{R})$ generators (44),

$$
\epsilon_{(t)}(z)=\left(\tau^{2}+\tau^{3}\right) \delta t \equiv \tau^{+} \delta t, \quad \bar{\epsilon}_{(t)}(\bar{z})=\left(\tau^{2}-\tau^{3}\right) \delta t \equiv-\tau^{-} \delta t .
$$

A similar calculation can be performed for the infinitesimal transformations in the $x$-direction, $\delta_{(x)} g$. Using the Ward identity (6) and substituting in $\epsilon$ and $\bar{\epsilon}$, it can then be seen that

$$
Q_{t}=\left(\mathcal{J}_{0}^{+}+\overline{\mathcal{J}}_{0}^{-}\right), \quad Q_{x}=\left(-\mathcal{J}_{0}^{+}+\overline{\mathcal{J}}_{0}^{-}\right) .
$$


In terms of the modified currents, the charges are then

$$
Q_{t}=\left(J_{0}^{+}+\bar{J}_{0}^{+}\right), \quad Q_{x}=-\left(J_{0}^{+}-\bar{J}_{0}^{+}\right) .
$$

We obtain the $M=0$ BTZ black hole by making periodic identifications along the $\partial_{x}$ direction. The period of the identification can be changed by rescaling $x$, so it is not a physical parameter. For convenience, we choose $x \sim x+2 \pi$. Invariance under this orbifold restricts states to have a quantised value of $Q_{x}$,

$$
\left(J_{0}^{+}-\bar{J}_{0}^{+}\right) \in \mathbb{Z}
$$

We therefore need to work in a parabolic basis for $S L(2, \mathbb{R})$, which diagonalises $J_{0}^{+}$. In the next section, we will use a Wakimoto representation for these currents to implement this constraint.

\section{Vertex operators on zero mass black hole}

In this section, we construct vertex operators for the untwisted and twisted sector states of the bosonic string on the $M=0$ BTZ black hole.

To implement the constraint (25), it is crucial to have a set of vertex operators which diagonalise the action of $J_{0}^{+}$. It is therefore useful to have a representation of the current algebra where $J^{+}$is as simple as possible. In the case of non-extreme BTZ black hole in [8], we needed a representation which diagonalised $J^{2}$, and we could simply introduce a free boson representing the current $J^{2}$, writing the remainder of the vertex operator in terms of a parafermion. Since the current $J^{+}$is null, a simple free boson representation will not be possible. However, it turns out that the hyperbolic Wakimoto representation of the $S \widehat{L(2, \mathbb{R})}$ current algebra introduced in 4 provides a simple representation for $J^{+}$(the relevance of this representation for the $M=0$ BTZ black hole was previously noted in [1]). The Wakimoto representation constructs the conserved currents in terms of a free boson $\phi$ and anticommuting $\beta-\gamma$ bosonic ghosts:

$$
\begin{aligned}
& i J^{+}(z)=\beta(z), \\
& i J^{-}(z)=\gamma^{2} \beta+\sqrt{2 k^{\prime}} \gamma \partial \phi(z)+k \partial \gamma(z), \\
& i J^{1}(z)=-\gamma \beta(z)-\sqrt{\frac{k^{\prime}}{2}} \partial \phi(z),
\end{aligned}
$$

where $k^{\prime} \equiv k-2$, and the OPEs for $\beta, \gamma$ and $\phi$ are

$$
\begin{aligned}
\beta(z) \gamma(w) & =-\gamma(z) \beta(w) \sim \frac{1}{z-w}, \\
\phi(z) \phi(w) & \sim-\ln (z-w)
\end{aligned}
$$


This leads to the required OPEs for the conserved currents,

$$
\begin{aligned}
J^{+}(z) J^{-}(w) & \sim \frac{-k}{(z-w)^{2}}+\frac{2 i J^{1}(w)}{z-w} \\
J^{1}(z) J^{ \pm}(w) & \sim \frac{\mp i J^{ \pm}(w)}{z-w} \\
J^{1}(z) J^{1}(w) & \sim \frac{\frac{k}{2}}{(z-w)^{2}}
\end{aligned}
$$

Unlike in the non-zero mass BTZ black hole case, this is an explicit representation of the full current algebra. We introduce an identical representation for the antiholomorphic currents $\bar{J}^{a}$, in terms of $\bar{\beta}(\bar{z}), \bar{\gamma}(\bar{z}), \bar{\phi}(\bar{z})$.

\subsection{Untwisted sector vertex operators}

We want to take a basis of vertex operators which diagonalise $J_{0}^{+}$. A vertex operator $V$ has $J_{0}^{+}$eigenvalue $\lambda$ if $J^{+} V(z)=\frac{\lambda V(z)}{z-w}$. Using (29), this implies that

$$
V(z)=e^{i \lambda \gamma} f(\beta, \phi) .
$$

For $\mathrm{AdS}_{3}$, the $S \widehat{L(2, \mathbb{R})}$ current algebra is a spectrum generating algebra, and the spectrum contains short string states in highest weight representations of the current algebra: the continuous representations $\hat{\mathcal{C}}_{j}^{\alpha} \times \hat{\mathcal{C}}_{j}^{\alpha}$ for $j=\frac{1}{2}+i s$ which correspond to spacetime tachyons, and the discrete representations $\hat{\mathcal{D}}_{j}^{ \pm} \times \hat{\mathcal{D}}_{j}^{ \pm}$for $\frac{1}{2}<j<\frac{k-1}{2}$. The spectrum in global AdS 3 also contains long string states, but these do not survive the orbifold projection, being replaced instead by the twisted sector states. Diagonalising $J_{0}^{+}$corresponds to considering the representations of $S L(2, \mathbb{R})$ in a parabolic basis. For both the continuous and discrete representations of the current algebra, in this parabolic representation, the eigenvalue $\lambda$ can take all real values.

Since these are highest weight representations of the current algebra, we can focus on the chiral primary operators; other vertex operators will be obtained as descendents. Requiring that (34) be a chiral primary operator implies that $f(\beta, \phi)$ is independent of $\beta$, as $V$ would otherwise have too singular an OPE with $J^{-}$. Including the anti-holomorphic sector, we can therefore take a basis of chiral primary vertex operators in the untwisted sector of the form

$$
V_{j \lambda \bar{\lambda}}(z, \bar{z})=e^{i \lambda \gamma-\sqrt{\frac{2}{k^{\prime}}} j \phi} e^{i \bar{\lambda} \bar{\gamma}-\sqrt{\frac{2}{k^{\prime}}} j \bar{\phi}} .
$$

To implement the orbifold, we need to quantise the eigenvalue of $J_{0}^{+}-\bar{J}_{0}^{+}$, that is, we need

$$
\lambda-\bar{\lambda} \in \mathbb{Z}
$$

In the next subsection, we will see how this quantisation condition can be implemented using a twist operator. This will also allow us to construct vertex operators for the twisted sector modes.

The energy momentum tensor for the WZW model is

$$
T=\frac{1}{k-2} \eta_{a b}: J^{a} J^{b}:
$$


which can be rewritten in in terms of $J^{+}$and $J^{-}$as

$$
T=\frac{1}{(k-2)}:\left(J^{1} J^{1}-\frac{1}{2} J^{+} J^{-}-\frac{1}{2} J^{-} J^{+}\right):
$$

Working in the Wakimoto representation,

$$
T=\beta \partial \gamma-\frac{\partial^{2} \phi}{\sqrt{2 k^{\prime}}}-\frac{(\partial \phi)^{2}}{2} .
$$

The conformal dimensions of the vertex operators (35) are then

$$
h=\bar{h}=\frac{-j(j-1)}{(k-2)} .
$$

Thus, the label $j$ on the vertex operators corresponds to the label on representations of the current algebra.

\subsection{Twist operator}

So far, we have just described the vertex operators describing strings on $\mathrm{AdS}_{3}$ in a basis which is adapted to working in Poincaré coordinates. To describe the $M=0$ BTZ black hole, we would now like to impose the quantisation condition (25). Following the same route as in the analysis of the non-extremal BTZ black hole [8], we would like to impose this condition by requiring mutual locality of the untwisted sector vertex operators (35) with an appropriate twist operator. The twisted sector vertex operators will then be obtained by closure of the OPE including the twist operator.

To do this we have to change our representation again and bosonize the $\beta-\gamma$ system as in [4,

$$
\begin{aligned}
& \beta=\partial \phi_{+}, \\
& \gamma=\phi_{-},
\end{aligned}
$$

where

$$
\begin{gathered}
\phi_{ \pm}=\frac{1}{\sqrt{2}}\left(\phi_{0} \pm \phi_{1}\right), \\
\phi_{i}(z) \phi_{j}(w) \sim-\eta_{i j} \ln (z-w), \quad \eta_{i j}=\operatorname{diag}(-1,1) .
\end{gathered}
$$

We introduce a similar bosonization for $\bar{\beta}, \bar{\gamma}$. In this representation, the untwisted sector chiral primary fields become

$$
V_{j \lambda \bar{\lambda}}(z, \bar{z})=e^{i \lambda \phi_{-}-\sqrt{\frac{2}{k^{\prime}}} j \phi} e^{i \bar{\lambda} \bar{\phi}_{-}-\sqrt{\frac{2}{k^{\prime}} j \bar{\phi}}}
$$

This representation was used to discuss strings on $\mathrm{AdS}_{3}$ in Poincaré coordinates in [20]. It was noted there that there are potential logarithmic branch cuts associated with the definition of the boson $\phi_{+}$. We will now see (as also noted in [11]) that these branch cuts are correctly interpreted as reflecting winding around the $x$ direction, describing the twisted sectors in the string on the $M=0$ BTZ black hole. 
The appropriate twist operators are

$$
t_{n}=e^{i n\left(\phi_{+}+\bar{\phi}_{+}\right)}
$$

This will impose the correct quantisation condition, as

$$
t_{n}(z) V_{j \lambda \bar{\lambda}}(w) \sim \frac{\exp \left(i n \phi_{+}+i \lambda \phi_{-}-\sqrt{\frac{2}{k^{\prime}}} j \phi\right)}{(z-w)^{n \lambda}} \frac{\exp \left(i n \bar{\phi}_{+}+i \bar{\lambda} \bar{\phi}_{-}-\sqrt{\frac{2}{k^{\prime}}} j \bar{\phi}\right)}{(\bar{z}-\bar{w})^{n \bar{\lambda}}}
$$

so the OPE will only be mutually local for $\lambda-\bar{\lambda} \in \mathbb{Z}$.

We can also read off the twisted sector vertex operators from this OPE, obtaining

$$
V_{j n \lambda \bar{\lambda}}=\exp \left(i n \phi_{+}+i \lambda \phi_{-}-\sqrt{\frac{2}{k^{\prime}}} j \phi\right) \exp \left(i n \bar{\phi}_{+}+i \bar{\lambda} \bar{\phi}_{-}-\sqrt{\frac{2}{k^{\prime}}} j \bar{\phi}\right) .
$$

Note that the current algebra generators $J^{-}, J^{2}$ will have non-trivial monodromies around a twisted sector vertex operator because of the dependence on $\phi_{+}$, reflecting the twisting. The conformal dimensions for these twisted sector operators are

$$
h=-\frac{j(j-1)}{k^{\prime}}-n \lambda, \quad \bar{h}=-\frac{j(j-1)}{k^{\prime}}-n \bar{\lambda} .
$$

The level-matching condition $h-\bar{h} \in \mathbb{Z}$ is satisfied as a consequence of the quantisation of the charge $Q_{x}$ in (36). Comparing this to the spectrum obtained for the twisted sectors of the non-extreme BTZ black hole in [8], we see that the spectrum there reduces to this one in the limit $r_{+} \rightarrow 0$, as expected, with $r_{+} \lambda_{\text {there }}=-\lambda_{\text {here }}, r_{+} \bar{\lambda}_{\text {there }}=\bar{\lambda}_{\text {here }}$. The full vertex operators involve taking descendents of these chiral primary operators, and include a contribution from the internal CFT. The physical state conditions will then be

$$
-\frac{j(j-1)}{k^{\prime}}-n \lambda+h_{i n t}+N=-\frac{j(j-1)}{k^{\prime}}-n \bar{\lambda}+\bar{h}_{i n t}+\bar{N}=1,
$$

where $h_{i n t}, \bar{h}_{i n t}$ are the dimensions of the operator from the internal CFT, and $N, \bar{N}$ are oscillator numbers for the current algebra. We assume that the internal CFT is unitary, so $h_{\text {int }}, \bar{h}_{\text {int }} \geq 0$.

This is one of the main results of our paper: by adopting this bosonised version of the Wakimoto representation for the currents, we see that we can give completely explicit expressions for the vertex operators for the full spectrum of string states on the $\mathrm{M}=0 \mathrm{BTZ}$ black hole, including twisted sector states. In [11], this representation of the currents was also used to construct the Virasoro generators associated with the asymptotic isometries of the spacetime in terms of the worldsheet currents. This makes it possible to study the relation of the worldsheet theory to the dual CFT in AdS/CFT, as was done for global $\mathrm{AdS}_{3}$ in [21, 22].

\subsection{Tachyons in twisted sectors}

As we are working with the bosonic string, we know there is a tachyonic ground state in the untwisted sectors. We would like to see if there is a tachyon in the twisted sectors. In 
the extension to the supersymmetric case, the expectation is that there will be a choice of GSO projection which eliminates the ground state in the untwisted sectors but retains it in the odd twisted sectors (corresponding to choosing an antiperiodic spin structure on the orbifold circle in spacetime). Given the appearance of a tachyon in twisted sectors for the non-extreme BTZ black hole with $\sqrt{k} r_{+}<2$ [8], we would expect that there will be one here as well.

We need to consider carefully the definition of a tachyon. Classically, a spacetime field is tachyonic if it has normalisable solutions which grow exponentially in time. It is difficult to look directly for modes which grow exponentially in time from the worldsheet point of view, as this would require complex $\lambda, \bar{\lambda}$, which makes it difficult to see how we can satisfy the physical state condition $h=\bar{h}=1$ in the twisted sectors. We will therefore look instead for modes with zero energy; if a spacetime field has a normalisable solution of zero energy, it should generically also have solutions which grow exponentially in time, by continuity 2 Since the $x$ direction is spacelike everywhere in the $M=0$ BTZ black hole, it is physically reasonable to further restrict to modes which also have zero momentum along $x$; these should be the most tachyonic modes for a field with a given mass-squared. Thus, it suffices for us to consider modes with $\lambda=\bar{\lambda}=0$.

Thus, we are looking for normalisable modes with $\lambda=\bar{\lambda}=0$, satisfying the physical state condition. In this case, the physical state condition for the twisted sectors is identical to that in the corresponding untwisted sector, and the twisted sector states will satisfy the physical state condition whenever the corresponding untwisted sector states do. In particular, there are physical states obtained by spectral flow from the tachyon in the untwisted sector, which have $j(j-1)<0$.

The remaining condition is normalisability. In the untwisted sector, we know that we have the usual bosonic string tachyon, which has normalisable solutions with $\lambda=\bar{\lambda}=0$. The twist operator is expressed in terms of the Wakimoto representation, and not in terms of the spacetime coordinates, so it is not possible to rigorously relate the normalisability of twisted sector modes to the corresponding untwisted sector ones, but we expect that at least for large $k$, the twisting will not significantly modify the dependence on the radial coordinate, so twisted sector modes will be normalisable if the corresponding untwisted sector mode is. Thus, we expect that the $\lambda=\bar{\lambda}=0$ vertex operators in twisted sectors obtained from the tachyon in the untwisted sector will be normalisable, and hence give modes of a tachyon in the twisted sectors.

As in [8], these modes have roughly the same radial behaviour as for the untwisted sector tachyon, so they are not well-localised in the neighbourhood of the horizon. The twisted sector states are essentially long string states, which can propagate to the asymptotic boundary of the $M=0$ BTZ black hole at low cost in energy because of the coupling the NSNS 2-form field.

The expectation is that while the untwisted sector tachyons are removed by the GSO projection in the supersymmetric theory, the untwisted sector tachyons will remain. We will comment on this again when we discuss the extension of our work to the superstring later.

\footnotetext{
${ }^{2}$ In a black hole background, modes supported close to the horizon have low energy as a result of the gravitational redshift, so a non-tachyonic field will have an energy spectrum starting from zero. However, it will not have a normalisable mode of strictly zero energy, so we believe this condition is still physically appropriate even in the presence of a black hole horizon.
} 


\section{The Null orbifold limit}

An important source of intuition and a useful check on the calculations in studying orbifolds of $\mathrm{AdS}_{3}$ is to consider the limit $k \rightarrow \infty$, in which the space becomes flat. For the nonzero mass BTZ black hole studied in [8], there were two flat space limits of interest, the near horizon limit which focused on the region near the event horizon, and the Milne limit, which focused on the singularity. For the $M=0$ BTZ black hole the event horizon and the singularity are at the same point in space, $z=\infty$ in the coordinates of (12). The two limits are therefore replaced by one, the null orbifold limit. In this section, we consider the behaviour of the untwisted and twisted sector states we constructed above as we take this limit. This limit is most closely analogous to the Milne limit in [8].

To show that the $M=0$ BTZ black hole reduces to the null orbifold as we take $k \rightarrow \infty$ focusing on the region near the Poincaré horizon at $z=\infty$, we need to make a change of coordinates. If we define new coordinates

$$
y^{+}=\frac{\sqrt{k}}{z}, \quad y^{-}=\sqrt{k}(t+z), \quad y=x,
$$

then the metric (12) becomes

$$
d s^{2}=-\frac{\left(y^{+}\right)^{2}\left(d y^{-}\right)^{2}}{k}-2 d y^{+} d y^{-}+\left(y^{+}\right)^{2} d y^{2} .
$$

Taking the limit $k \rightarrow \infty$ for fixed $y^{ \pm}, y$, the first term vanishes, and we can see that the metric reduces to the null orbifold of [17, 18], which was analysed in string theory in [23, 24]. In these coordinates, the null orbifold is simply the identification $y \sim y+2 \pi$. The quantization condition associated with this orbifold identification remains simply $\lambda-\bar{\lambda} \in \mathbb{Z}$. We should also consider the limit for the NSNS 2-form field (13). To obtain a finite limit as $k \rightarrow \infty$, we first need to make a gauge transformation to write

$$
B=\frac{k}{z^{2}}(d t+d z) \wedge d x=\frac{\left(y^{+}\right)^{2}}{\sqrt{k}} d y^{-} \wedge d y,
$$

so in this gauge the 2 -form vanishes in the limit as $k \rightarrow \infty$. The contribution from the 2 -form is still important to see that the currents $J^{a}, \bar{J}^{a}$ are conserved to sub-leading order as we take the limit, as in [8].

It is also convenient to rewrite the null orbifold in Cartesian coordinates; the relation is

$$
x^{+}=y^{+}, \quad x^{-}=y^{-}+\frac{1}{2} y^{+} y^{2}, \quad x=y^{+} y .
$$

In these coordinates, the null orbifold metric is simply flat, but the identification is more complicated:

$$
\left(x^{+}, x^{-}, x\right) \sim\left(x^{+}, x^{-}+2 \pi x+2 \pi^{2} x^{+}, x+2 \pi x^{+}\right) .
$$


Using (14) the currents can be calculated in terms of $y^{ \pm}, y$ :

$$
\begin{aligned}
J^{1}= & -i \sqrt{k}\left(y^{+} \partial y+y \partial y^{+}\right)-i y^{+} \partial y^{-}+i y^{-} \partial y^{+}-i\left(y^{+}\right)^{2} y \partial y+O\left(\frac{1}{\sqrt{k}}\right), \\
J^{2}= & -i \sqrt{k}\left(y^{+} y \partial y+\partial y^{-}+\frac{y^{2} \partial y^{+}}{2}-\frac{\partial y^{+}}{2}\right) \\
& +i\left(-\frac{\left(y^{+}\right)^{2} y^{2} \partial y}{2}+y^{+} y^{-} \partial y+\frac{\left(y^{+}\right)^{2} \partial y}{2}-y^{+} y \partial y^{-}+y^{-} y \partial y^{+}\right)+O\left(\frac{1}{\sqrt{k}}\right), \\
J^{3}= & i \sqrt{k}\left(y^{+} y \partial y+\partial y^{-}+\frac{y^{2} \partial y^{+}}{2}+\frac{\partial y^{+}}{2}\right) \\
& -i\left(-\frac{\left(y^{+}\right)^{2} y^{2} \partial y}{2}+y^{+} y^{-} \partial y-\frac{\left(y^{+}\right)^{2} \partial y}{2}-y^{+} y \partial y^{-}+y^{-} y \partial y^{+}\right)+O\left(\frac{1}{\sqrt{k}}\right) .
\end{aligned}
$$

This can be more simply re-expressed in terms of $x^{ \pm}, x$ :

$$
\begin{aligned}
J^{1} & =-i \sqrt{k} \partial x-i x^{+} \partial x^{-}+i x^{-} \partial x^{+}+O\left(\frac{1}{\sqrt{k}}\right), \\
J^{2} & =-i \sqrt{k}\left(\partial x^{-}-\frac{\partial x^{+}}{2}\right)+i\left(x^{-} \partial x-x \partial x^{-}+\frac{1}{2}\left(x^{+} \partial x-x \partial x^{+}\right)\right)+O\left(\frac{1}{\sqrt{k}}\right), \\
J^{3} & =i \sqrt{k}\left(\partial x^{-}+\frac{\partial x^{+}}{2}\right)-i\left(x^{-} \partial x-x \partial x^{-}-\frac{1}{2}\left(x^{+} \partial x-x \partial x^{+}\right)\right)+O\left(\frac{1}{\sqrt{k}}\right) .
\end{aligned}
$$

We see that in the flat space limit $k \rightarrow \infty$, the leading order $(O(\sqrt{k}))$ terms reproduce the Cartesian translation currents on flat space. As in [8, the subleading parts involve Lorentz transformations in the flat space limit, and are required to make the total current conserved to subleading order taking into account the effects of the 2 -form field. The same can be done for the antiholomorphic sector.

We can use this expression for the currents to relate the Wakimoto variables to the coordinates in this limit,

$$
\begin{aligned}
\beta & =-\sqrt{k} \partial x^{+}-\left(x^{+} \partial x-x \partial x^{+}\right), \\
\gamma & =\frac{2}{\sqrt{k}} x^{-}+\frac{2}{k} x x^{-}, \\
\phi & =-\sqrt{\frac{2}{k^{\prime}}}\left(\sqrt{k} x+x^{+} x^{-}\right) .
\end{aligned}
$$

This in turn implies that the bosonised versions of the Wakimoto variables are closely related to the $x^{ \pm}, x$ coordinates in the flat space limit; to leading order,

$$
\phi^{+}=-\sqrt{k} x^{+}, \quad \phi^{-}=\frac{2}{\sqrt{k}} x^{-}, \quad \phi=-\sqrt{2} x .
$$

By studying the flat space limit of the antiholomorphic currents $\bar{J}^{a}$, one can similarly learn that

$$
\bar{\phi}^{+}=-\sqrt{k} \bar{x}^{+}, \quad \bar{\phi}^{-}=-\frac{2}{\sqrt{k}} \bar{x}^{-}, \quad \bar{\phi}=\sqrt{2} \bar{x}
$$

to leading order. Note that the factors of 2 in these expressions appear because in units with $\alpha^{\prime}=1$, the flat space coordinates have OPEs $x^{\mu} x^{\nu} \sim \frac{1}{2} \eta^{\mu \nu} \ln (z-w)$. 


\subsection{States and vertex operators}

In the untwisted sector, the states which survive in this flat space limit are those with $\lambda-\bar{\lambda} \in \mathbb{Z} \sim \mathcal{O}(1)$ and $\lambda+\bar{\lambda} \sim \mathcal{O}(\sqrt{k})$ (since we hold $y^{-}=\sqrt{k}(t+r)$ fixed in the limit). To have $h \sim \mathcal{O}(1)$, we need $j \sim \mathcal{O}(\sqrt{k})$, and similarly for the barred quantities. In the twisted sectors, we still have $\lambda-\bar{\lambda} \in \mathbb{Z} \sim \mathcal{O}(1)$ and $\lambda+\bar{\lambda} \sim \mathcal{O}(\sqrt{k})$, since the $Q_{x}$ and $Q_{t}$ eigenvalues are unaffected by twisting. However, in twisted sectors $h=-\frac{j(j-1)}{k-2}-n \lambda$, so $h \sim \mathcal{O}(1)$ in twisted sectors requires $j \sim \mathcal{O}\left(k^{3 / 4}\right)$ to cancel the $\sqrt{k}$ contribution from $\lambda$. This cancellation can be achieved for modes in the continuous representations of the current algebra if $\lambda>0$, and for modes in the discrete representations of the current algebra if $\lambda<0$. Thus, both tachyonic and non-tachyonic twisted sector modes survive in the flat space limit, but with this curious correlation with the sign of $\lambda$. We expect that the resulting spectrum in the flat space limit should agree with the one obtained in [23].

We note that as in the Milne limit in [8], in general the modes which survive in twisted sectors in this flat space limit are not the ones which are obtained by twisting from the untwisted sector states which survive in the limit. This can also be seen by observing that the twist operator (46) becomes, to leading order,

$$
t_{n}=e^{-n \sqrt{k}\left(x_{+}+\bar{x}_{+}\right)},
$$

and hence does not have a well-defined flat space limit. Thus, our twist operator construction does not have a counterpart in the null orbifold.

Despite the failure of the twist operator to survive in the flat space limit, one might still hope that we could follow our vertex operators in this limit, since we have an explicit construction of the vertex operators in terms of the Wakimoto representation and we understand how these Wakimoto fields are related to flat space coordinates in the limit. Disappointingly, this does not work. If we consider the vertex operator (35) and substitute in the leading order relations between the Wakimoto fields and the coordinates (65), we obtain

$$
V_{j \lambda \bar{\lambda}}(z)=e^{i \frac{2 \lambda}{\sqrt{k}} x^{-}+j \frac{2 \sqrt{k}}{k-2} x} e^{-i \frac{2 \bar{\lambda}}{\sqrt{k}} \bar{x}^{-}-j \frac{2 \sqrt{k}}{k-2} \bar{x}}
$$

In the limit, let us write $2 \lambda=\sqrt{k} E+P_{x}, 2 \bar{\lambda}=\sqrt{k} E-P_{x}, j=\sqrt{k} m$. Then the vertex operator is to leading order

$$
V_{j \lambda \bar{\lambda}}(z)=e^{i E x^{-}+2 m x} e^{-i E \bar{x}^{-}-2 m \bar{x}} .
$$

We see that this expression has lost the dependence on $P_{x}$, so it degenerates in the flat space limit. This failure to obtain a good representation of the vertex operators in the flat space limit is in retrospect not unexpected: the $S \widehat{L(2, \mathbb{R})} \times S \widehat{L(2, \mathbb{R})}$ structure we used in constructing our vertex operators degenerates in this limit. Although the metric and spectrum smoothly go over to the flat space orbifold in this limit, a new representation of the vertex operators is necessary. Similarly, when we take the $r_{+} \rightarrow 0$ limit of the nonzero mass BTZ black hole, the spectrum of [8] reduces to the one we have obtained here for the zero-mass black hole, but the vertex operators do not have a smooth limit. A new representation is needed in the limit, reflecting the fact that we are considering a different orbifold. 
Thus, while the orbifold we are considering reduces to the null orbifold in the limit $k \rightarrow \infty$, the representation of the vertex operators in terms of Wakimoto fields degenerates in this limit, so we do not seem to be able to glean new insight into the null orbifold from our construction.

\section{The extremal rotating black hole}

We can easily extend this investigation of the zero mass BTZ black hole to study extremal rotating BTZ black holes, with $M=J$. Rotating BTZ black holes are orbifolds of $\mathrm{AdS}_{3}$ with an asymmetric action on the worldsheet; as we will see below, the extremal rotating black holes correspond to an orbifold where the action on the left-movers is the same as for the zero mass BTZ black hole, while the action on the right movers is the same as for the non-zero mass black hole studied in [8]. Thus, by combining our previous results, it is easy to determine the spectrum in this case as well. The construction of the twisted sectors is based on an appropriate ansatz, for which we then explicitly check the mutual locality.

The extremal rotating BTZ black hole has the metric

$$
d s^{2}=k\left(-\frac{\left(r^{2}-r_{+}^{2}\right)^{2}}{r^{2}} d \tau^{2}+\frac{r^{2}}{\left(r^{2}-r_{+}^{2}\right)^{2}} d r^{2}+r^{2}\left(d \phi-\frac{r_{+}^{2}}{r^{2}} d t\right)^{2}\right),
$$

where $\phi$ is a periodic coordinate, $\phi \sim \phi+2 \pi$. The spacetime is locally $\mathrm{AdS}_{3}$, with periodicity of $\phi$ corresponding to the action of an orbifold. If we write $\mathrm{AdS}_{3}$ in the embedding coordinates $x^{0}, x^{1}, x^{2}, x^{3}$ in $\mathbb{R}^{2,2}$ as the hyperboloid $-x_{0}^{2}-x_{1}^{2}+x_{2}^{2}+x_{3}^{3}=-k^{2}$, the orbifold which gives us the above extremal rotating black hole is along the Killing vector [2]

$$
\xi=r_{+}\left(J_{03}+J_{12}\right)+J_{01}-J_{02}-J_{13}+J_{23},
$$

up to conjugation, where $J_{a b}$ are the Lorentz transformations on $\mathbb{R}^{2,2}, J_{a b}=\eta_{b c} x^{c} \partial_{a}-\eta_{a c} x^{c} \partial_{b}$. In the coordinate system of (70), this Killing vector is $\xi=\partial_{\phi}$.

The extremal rotating BTZ metric is locally $\mathrm{AdS}_{3}$, so it can be related to the Poincaré coordinate system we used earlier. The coordinate transformation

$$
z=R^{-1 / 2} e^{r_{+}(\phi-t)}, \quad t+x=e^{2 r_{+}(\phi-t)}, \quad t-x=-(T+\phi),
$$

where

$$
R=\frac{1}{2 r_{+}}\left(r^{2}-r_{+}^{2}\right), \quad T=t-\frac{r_{+}}{r^{2}-r_{+}^{2}},
$$

converts the metric (12) into the metric (70). This corresponds to writing the group element of $S L(2, \mathbb{R})$ as

$$
g=\left(\begin{array}{cc}
R^{\frac{1}{2}} e^{-r_{+}(\phi-t)} & R^{\frac{1}{2}} e^{r_{+}(\phi-t)} \\
(T+\phi) R^{\frac{1}{2}} e^{-r_{+}(\phi-t)} & R^{\frac{1}{2}} e^{r_{+}(\phi-t)}\left(T+\phi+\frac{1}{R}\right)
\end{array}\right) .
$$

As in section 2.1, we can determine the conserved charge associated with $\phi$ translation. The action of $\phi$ translation on the group element is

$$
\delta_{(\phi)} g=\left(\begin{array}{ll}
0 & 0 \\
1 & 0
\end{array}\right) g+r_{+} g\left(\begin{array}{cc}
-1 & 0 \\
0 & 1
\end{array}\right)
$$


so the conserved charge is

$$
Q_{\phi}=-J_{0}^{+}+r_{+} \bar{J}_{0}^{1} .
$$

Note that this naive expression will apply for the untwisted sectors; for the twisted sectors, there is the possibility of a total derivative term, which we need to determine.

Thus, we see that the action in terms of $S L(2, \mathbb{R}) \times S L(2, \mathbb{R})$ is chiral, with the left moving part looking like that of a massless BTZ black hole we have studied above, while the right moving part looks like that of the massive BTZ black hole. It is therefore natural to choose the parafermionic representation for the right movers, and the Wakimoto representation introduced above for the left movers. The parafermionic representation for the right movers involves writing the currents as

$$
\bar{J}^{1}=-i \sqrt{\frac{k}{2}} \partial \bar{X}, \quad \bar{J}^{ \pm}=\bar{\xi}^{ \pm} e^{ \pm \sqrt{\frac{2}{k}} \bar{X}}
$$

where $\bar{X}$ is a free boson, $\bar{X}(\bar{z}) \bar{X}(\bar{w}) \sim-\ln (\bar{z}-\bar{w})$, and $\bar{\xi}^{ \pm}$are parafermions representing the remaining $S \widehat{L(2, \mathbb{R})_{k}} / \widehat{U(1)}$ algebra. Thus, the vertex operators in the untwisted sector are

$$
V_{j \lambda \bar{\lambda}}(z)=e^{i \lambda \gamma-j \sqrt{\frac{2}{k^{\prime}}} \phi} \bar{\Psi}_{j \bar{\lambda}} e^{-i \sqrt{\frac{2}{k}} \bar{\lambda} \bar{X}}
$$

Where $\bar{\Psi}_{j \bar{\lambda}}$ are parafermionic operators with conformal dimension $\bar{h}_{\bar{\Psi}}=-\frac{j(j-1)}{k^{\prime}}-\frac{\bar{\lambda}^{2}}{k}$. We know that these untwisted sector vertex operators are mutually local for the operators corresponding to modes of fields on AdS. This gives us some information about the OPE of the parafermionic operators, as the OPE of two such vertex operators will only be mutually local if

$$
m-\frac{2}{k^{\prime}} j j^{\prime}-\frac{2}{k} \bar{\lambda} \bar{\lambda}^{\prime} \in \mathbb{Z}
$$

where $m$ characterises the leading singularity in the OPE of the parafermionic operators,

$$
\bar{\Psi}_{j \bar{\lambda}} \bar{\Psi}_{j^{\prime} \bar{\lambda}^{\prime}} \sim \frac{O}{(z-w)^{m}} .
$$

We construct an ansatz for the twisted sector states in this orbifold by combining the results for the twisted sectors from our earlier analysis of the massive and massless black holes: that is, we guess that the twisted sector vertex operators are simply the right moving part of the twisted sector state from the massive black hole, combined with the left moving part of the twisted sector state from the massless black hole. This gives

$$
V_{j \lambda \bar{\lambda} n}(z)=\exp \left(i n \phi_{+}+i \lambda \phi_{-}-j \sqrt{\frac{2}{k^{\prime}}} \phi\right) \bar{\Psi}_{j \bar{\lambda}} \exp \left(-i \sqrt{\frac{k}{2}}\left[\bar{\lambda}-\frac{k}{2} n r_{+}\right] \bar{X}\right)
$$

The conformal dimensions for this operator are

$$
h=-\frac{j(j-1)}{k^{\prime}}-n \lambda, \quad \bar{h}=-\frac{j(j-1)}{k^{\prime}}-\bar{\lambda} r_{+} n+\frac{k n^{2} r_{+}^{2}}{4} .
$$

As in section 3.3, we should have a tachyon in the twisted sectors if we can satisfy the physical state condition for a mode with $j(j-1)<0$ and $\lambda=\bar{\lambda}=0$. This requires $\sqrt{k} r_{+}<2$, as 
in [8], so there is a tachyon if the size of the spatial circle at the black hole horizon is small enough. As in section [3.3, this tachyon will not be well localised in the region near the horizon.

Level matching will require that $h-\bar{h} \in \mathbb{Z}$, which implies

$$
-n \lambda+r_{+} n \bar{\lambda}-\frac{k n^{2} r_{+}^{2}}{4} \in \mathbb{Z} .
$$

We would like to see this arise as a consequence of the quantisation of angular momentum imposed by the orbifold. Naively, the generator of translation in $\phi$ is (176), which would imply a quantisation condition $-\lambda+r_{+}\left(\bar{\lambda}-k n r_{+} / 2\right) \in \mathbb{Z}$, which does not agree with (83). Hence, as in [5], we will need to include a total derivative term in the definition of $Q_{\phi}$, so that in twisted sectors

$$
Q_{\phi}=-J_{0}^{+}+r_{+} \bar{J}_{0}^{1}+\frac{k n r_{+}^{2}}{4},
$$

implying a quantization condition

$$
-\lambda+r_{+} \bar{\lambda}-\frac{k n r_{+}^{2}}{4} \in \mathbb{Z}
$$

consistent with (83). Note that unlike in non-rotating cases, the quantization condition on $\lambda, \bar{\lambda}$ depends on the twist $n$, so the twisted sector states cannot be obtained by considering the OPE of untwisted sector states with an appropriate twist operator. Such a procedure would get the quantization condition wrong.

We then need to verify mutual locality of these twisted sector states. To determine mutual locality consider an OPE between $V_{j \lambda \bar{\lambda} n}(z)$ and $V_{j^{\prime} \lambda^{\prime} \bar{\lambda}^{\prime} n^{\prime}}(w)$. It turns out that the relevant condition for mutual locality is

$$
m-\frac{2}{k^{\prime}} j j^{\prime}-n \lambda^{\prime}-n \lambda-\frac{2}{k}\left(\bar{\lambda}-\frac{k}{2} n r_{+}\right)\left(\bar{\lambda}^{\prime}-\frac{k}{2} n^{\prime} r_{+}\right) \in \mathbb{Z}
$$

This is satisfied as a consequence of (83) and (779). Therefore the correct spectrum for the twisted sector is indeed given by (81); this completes the untwisted sector spectrum to obtain a mutually local set of operators with the appropriate set of twisted sectors, indexed by a single integer denoting the twist. It would be interesting to see if the modular invariance of the resulting partition function could be explicitly verified, but this will be complicated to check, as is generally the case for asymmetric orbifolds, so we will not attempt to do so explicitly here.

\section{Supersymmetry}

In this section, we will discuss the extension of our analysis of the spectrum to the superstring. This analysis is particularly interesting for the $M=J$ extremal rotating black holes considered here, as these are supersymmetric backgrounds for the superstring. Also, from the point of view of considering the tachyons, the tachyons in twisted sectors are most interesting in the superstring, where we expect the GSO projection to eliminate the tachyon in the 
untwisted sector. For the supersymmetric choice of spin structure on spacetime, spacetime supersymmetry implies that there are no tachyons, but if we make the opposite choice of spin structure, we expect the tachyons in odd twisted sectors to survive the GSO projection.

Unfortunately, we are not able to construct the spectrum for the superstring explicitly. We have not succeeded in extending the nice representation of $S L(2, \mathbb{R})$ in terms of Wakimoto fields to the superstring, so we have not succeeded even in constructing an explicit representation for the untwisted sector modes which survive the orbifold projection condition. In this section, we will describe the problem, focusing on the case of the $M=0$ black hole for simplicity.

We consider Type II String theory on BTZ $\times \mathbf{S}^{\mathbf{3}} \times \mathbf{T}^{\mathbf{4}}$ as described by a $S \widehat{L(2, \mathbb{R})}$ superWZW model at level $k$. The super-current algebra is

$$
J^{a}=j^{a}-\frac{i}{k} \epsilon_{b c}^{a} \psi^{b} \psi^{c}
$$

with $\psi^{a}$ having the usual OPE structure for a fermion,

$$
\begin{aligned}
\psi^{a}(z) \psi^{b}(w) & \sim \frac{k}{2} \frac{\eta^{a b}}{(z-w)} \\
j^{a}(z) \psi^{b}(w) & \sim 0
\end{aligned}
$$

The OPEs for the bosonic currents $j^{a}$ are almost identical to the previous section, while the OPEs for the supercurrent $J^{a}$ are very similar,

$$
\begin{aligned}
j^{a}(z) j^{b}(w) & \sim \frac{\tilde{k}}{2} \frac{\eta^{a b}}{(z-w)^{2}}+i \frac{\epsilon^{a b}{ }_{c} j^{c}}{(z-w)} \\
J^{a}(z) J^{b}(w) & \sim \frac{k}{2} \frac{\eta^{a b}}{(z-w)^{2}}+i \frac{\epsilon^{a b}{ }_{c} j^{c}}{(z-w)}
\end{aligned}
$$

where $\tilde{k}=k+2$. The world-sheet $\mathcal{N}=1$ super-current is

$$
G(z)=\frac{2}{k}\left(g_{a b} \psi^{a} j^{b}-\frac{i}{3 k} \epsilon_{a b c} \psi^{a} \psi^{b} \psi^{c}\right)
$$

For the superstring, the generator of $x$ translations is again (23), but where $J^{a}$ are now the total currents given in (87). We therefore want to find a representation of the total currents which simplifies the action of $J^{+}$, and use this representation to write the untwisted sector vertex operator in a form which diagonalises the action of $J^{+}$. We can use a Wakimoto representation as before for the bosonic currents $j^{a}$, but the fermionic contribution to $J^{a}$ is more problematic.

In [10, 8], the fermions were rewritten in terms of a set of bosons $H_{I}, I=1, \ldots, 5$, with OPEs

$$
H_{I}(z) H_{J}(w)=-\delta_{I J} \ln (z-w)
$$

such that the spin fields

$$
S_{\alpha}=e^{\frac{i}{2} \epsilon_{I} H_{I}}
$$


with $\epsilon_{I}= \pm 1$ diagonalise the action of $J^{2}$ and hence $Q_{\phi}$. In our case, it is $J^{+}$which is relevant, and this involves a combination $\psi_{1}\left(\psi_{3}+\psi_{2}\right) \equiv \psi_{1} \psi_{+}$. We could formally define a field $H_{*}$ by

$$
\partial H_{*}=\psi_{1} \psi_{+}
$$

but this will not have the OPE of a free boson, so we cannot use it in constructing the spin fields in a way analogous to [10, 8]. As a result, we have no simple route to constructing an appropriate basis of spin fields in this case which diagonalises the action of $Q_{x}$. We leave this as an open problem for future work. It is clearly very interesting to try to understand these simple examples of BPS black holes from the worldsheet perspective, so we hope further progress will be possible.

\section{Discussion}

The main result of this paper is that we have obtained the full spectrum and discussed the tachyons appearing in this spectrum for the bosonic string on the $M=0$ BTZ black hole and on the extremal rotating $M=J$ black hole. The spectrum on the zero mass black hole is just the limit of the spectrum obtained for the massive black hole in [8], as we would expect. However, because the zero mass black hole corresponds to a parabolic orbifold of $\mathrm{AdS}_{3}$, the description of the states in this case is quite different from that of [8]. The use of the Wakimoto representation in the $M=0$ BTZ black hole enables us to give a fully explicit description of the vertex operators for states in both untwisted and twisted sectors. This makes this example a particularly interesting laboratory for further explorations of worldsheet string theory on these black hole backgrounds; compared to the parafermionic representation of the vertex operators employed for the massive black hole in [8], this more explicit representation ought to give us greater control. Unfortunately, however, this description of the vertex operators appears to degenerate in the flat space limit, so our understanding of the limit where we zoom in on the region near the singularity is not significantly improved by the use of this representation.

For the zero mass black hole, we argued that the twisted sector modes obtained from the bulk tachyon in the untwisted sector are also tachyonic. The analysis of these tachyons will closely parallel the corresponding analysis in [8], so we have not given much detail in our discussion of the tachyons. These modes are not localised in the region near the horizon, as the coupling to the NSNS 2-form field makes a negative contribution to the energy of the string, allowing it to propagate to large distances. The study of the condensation of these tachyons would be an interesting direction for further work, but because the tachyon is not localised, it may be quite challenging.

We extended our work on the zero mass black hole by considering the extremal rotating black hole, which corresponds to an asymmetric orbifold, with the action on left movers the same as for the zero mass black hole and the action on right movers the same as for the massive non-rotating black hole. We were therefore able to construct a proposal for the spectrum of strings on this background by combining our work on the zero mass black hole with previous work on the massive black hole.

Finally, we considered the extension to the superstring. For the elliptic or hyperbolic orbifolds, it was possible to extend the orbifold construction to the superstring by choosing 
an appropriate set of spin fields which were eigenfunctions of the momentum along the compact direction, allowing us to construct superstring vertex operators which satisfy the appropriate quantisation condition. We were unable to find a corresponding basis for the parabolic orbifold which gives the $M=0$ BTZ black hole; as a result, we cannot construct superstring vertex operators which are well-defined on the orbifold spacetime. This technical problem appears to be the most important direction for further work: obviously, the main motivation for interest in the $M=0$ and $M=J$ black holes is that they are supersymmetric solutions in appropriate supergravity theories [25]. Also, the study of the tachyons in twisted sectors is mainly interesting in the context of the superstring, where we expect to be able to choose a GSO projection which will project out the untwisted sector tachyon but keep the tachyonic modes in odd twisted sectors. Any further progress on these directions will require an appropriate construction of vertex operators for the superstring.

\section{Acknowledgements}

We thank Mukund Rangamani for useful discussions. This work was supported by the STFC.

\section{References}

[1] M. Banados, C. Teitelboim, and J. Zanelli, "The Black hole in three-dimensional space-time," Phys. Rev. Lett. 69 (1992) 1849-1851, hep-th/9204099.

[2] M. Banados, M. Henneaux, C. Teitelboim, and J. Zanelli, "Geometry of the $(2+1)$ black hole," Phys. Rev. D 48 (1993) 1506-1525, gr-qc/9302012.

[3] M. Natsuume and Y. Satoh, "String theory on three dimensional black holes," Int. J. Mod. Phys. A13 (1998) 1229-1262, hep-th/9611041.

[4] Y. Satoh, "Ghost-free and modular invariant spectra of a string in $S L(2, R)$ and three-dimensional black hole geometry," Nucl. Phys. B513 (1998) 213-228, hep-th/9705208.

[5] S. Hemming and E. Keski-Vakkuri, "The spectrum of strings on BTZ black holes and spectral flow in the $S L(2, R)$ WZW model," Nucl. Phys. B626 (2002) 363-376, hep-th/0110252.

[6] E. J. Martinec and W. McElgin, "Exciting AdS orbifolds," JHEP 10 (2002) 050, hep-th/0206175.

[7] S. Hemming, E. Keski-Vakkuri, and P. Kraus, "Strings in the extended BTZ spacetime," JHEP 10 (2002) 006, hep-th/0208003.

[8] M. Rangamani and S. F. Ross, "Winding tachyons in BTZ," Phys. Rev. D 77 (2008) 026010, 0706.0663.

[9] R. Argurio, A. Giveon, and A. Shomer, "Superstrings on AdS(3) and symmetric products," JHEP 12 (2000) 003, hep-th/0009242. 
[10] E. J. Martinec and W. McElgin, "String theory on AdS orbifolds," JHEP 04 (2002) 029, hep-th/0106171.

[11] J. Troost, "Winding strings and AdS 3 black holes," JHEP 09 (2002) 041, hep-th/0206118.

[12] A. Adams, X. Liu, J. McGreevy, A. Saltman, and E. Silverstein, "Things fall apart: Topology change from winding tachyons," JHEP 10 (2005) 033, hep-th/0502021.

[13] G. T. Horowitz, "Tachyon condensation and black strings," JHEP 08 (2005) 091, hep-th/0506166.

[14] S. F. Ross, "Winding tachyons in asymptotically supersymmetric black strings," JHEP 10 (2005) 112, hep-th/0509066.

[15] J. McGreevy and E. Silverstein, "The tachyon at the end of the universe," JHEP 08 (2005) 090, hep-th/0506130.

[16] G. T. Horowitz and E. Silverstein, "The inside story: Quasilocal tachyons and black holes," Phys. Rev. D 73 (2006) 064016, hep-th/0601032.

[17] A. R. Steif, "Supergeometry of three-dimensional black holes," Phys. Rev. D 53 (1996) 5521-5526, hep-th/9504012.

[18] J. Simon, "The geometry of null rotation identifications," JHEP 06 (2002) 001, hep-th/0203201.

[19] J. M. Maldacena and H. Ooguri, "Strings in $\operatorname{AdS}(3)$ and SL(2,R) WZW model. I," J. Math. Phys. 42 (2001) 2929-2960, hep-th/0001053.

[20] I. Bars, "Ghost - free spectrum of a quantum string in $S L(2, R)$ curved space-time," Phys. Rev. D 53 (1996) 3308-3323, hep-th/9503205.

[21] A. Giveon, D. Kutasov, and N. Seiberg, "Comments on string theory on $\mathrm{AdS}_{3}$," Adv. Theor. Math. Phys. 2 (1998) 733-780, hep-th/9806194.

[22] J. de Boer, H. Ooguri, H. Robins, and J. Tannenhauser, "String theory on $\mathrm{AdS}_{3}$," JHEP 12 (1998) 026, hep-th/9812046.

[23] H. Liu, G. W. Moore, and N. Seiberg, "Strings in a time-dependent orbifold," JHEP 06 (2002) 045, hep-th/0204168.

[24] H. Liu, G. W. Moore, and N. Seiberg, "Strings in time-dependent orbifolds," JHEP 10 (2002) 031, hep-th/0206182.

[25] O. Coussaert and M. Henneaux, "Supersymmetry of the $(2+1)$ black holes," Phys. Rev. Lett. 72 (1994) 183-186, hep-th/9310194. 